\title{
Elucidating Jet Energy Loss Using Jets: Prospects from ATLAS
}

\author{
N. Grau ${ }^{a}$, on behalf of the ATLAS Collaboration \\ ${ }^{a}$ Columbia University, Nevis Laboratories, Irvington, NY 10533, USA
}

\begin{abstract}
Jets at the LHC are expected to provide the testing ground for studying QCD energy loss. In this contribution, we briefly outline the strategy that will be used to measure jets in ATLAS and how we will go about studying energy loss. We describe the utility of measuring the jet $R_{A A}$, the fragmentation function, and heavy flavor jets. Utilizing the collision energy provided by the LHC and the nearly hermetic and highly segmented calorimeter, ATLAS is expected to make important contributions to the understanding of parton energy loss using fully reconstructed jets.
\end{abstract}

\section{Introduction}

A wealth of measurements, interpreted as in terms of parton energy loss, have been made at the Relativistic Heavy Ion Collider (RHIC) in single particle measurements and multi-particle correlations [1] in A+A collisions. Even at this time the debate continues on the mechanism or energy loss being either perturbative in nature, i.e. gluon radiation, or some non-purturbative strong coupling phenomenon. New experimental measurements are necessary in order to shed light on the mechanism and behavior of QCD energy loss. Interestingly, new results from full jet reconstruction in heavy ion collisions at RHIC are becoming available [2]. In this contribution, we present the prospects for measuring fully reconstructed jets using the ATLAS detector at the LHC. We outline several differential studies that can be performed with fully reconstructed jets and how they will extend our knowledge of what has been inferred about energy loss from measurements at RHIC.

The ATLAS detector is a large multi-purpose apparatus designed to measure rare high- $p_{\mathrm{T}}$ phenomena in high energy hadronic collisions [3]. Though designed for $p+p$ collisions, the capability of the detector for tracking, calorimetry, and muon identification are more than sufficient for use even in the extreme particle densities expected at the LHC [4]. For jet reconstruction, only the calorimeter is used in these studies. The ATLAS calorimeter consists of an electromagnetic calorimeter covering $|\eta|<3.2$ and full azimuth. The electromagnetic calorimeter consists of three sampling layers of varying radiation length and varying cell (single readout channel) sizes. The hadronic calorimeter, also consisting of multiple sampling layers, covers $|\eta|<5.0$ and full azimuth. Such a calorimeter presents unprecedented coverage for measuring jets in nuclear collisions at collider energies.

For this contribution, we focus on results from the cone algorithm for jet reconstruction. The ATLAS cone algorithm is a seeded cone algorithm run on calorimeter "towers", sums of EM and hadronic energy in bins of $0.1 \times 0.1$ in $\eta \times \phi$ (position) space. There is a requirement for one tower to exceed some predefined threshold seeding the algorithm. For each seed tower, the 4 -vector sum of the towers within a cone in $\eta \times \phi$ space with radius $R$ is taken. The position of this 4-vector sum is then used as the new jet position. The 4-vector summing in a cone is iterated 


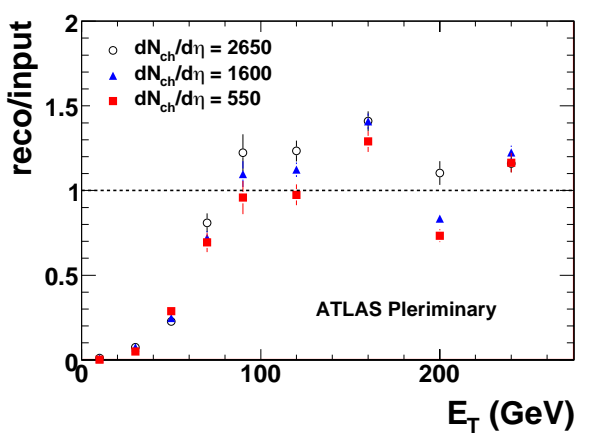

Figure 1: Ratio of reconstructed to input jet spectrum for PYTHIA jets embedded in HIJING events. The jets are reconstructed with seed $E_{\mathrm{T}}=10 \mathrm{GeV}$ and $R=0.4$. There are no corrections for efficiency, energy scale, or energy resolution.

until the 4-vector sum position converges. It is possible that multiple towers in a jet are above the seed threshold producing multiple jets with overlapping constituents. An overlap fraction, the fraction of constituent towers shared between a pair of jets, is used to determine whether to combine or separate multiple jets, such that each tower belongs to one and only one jet. The ATLAS cone algorithm is defined by three independent parameters: 1) the cone radius $R, 2$ ) the seed threshold, and 3) the overlap fraction.

In heavy ion collisions, jet reconstruction must be modified to handle the underlying event energy. We have chosen to determine the underlying event energy, remove it from the calorimeter towers, and then run the cone algorithm as described above. The background determination is as follows. First, high-energy regions of the calorimeter are identified by performing a sliding window algorithm which calculates the scalar $E_{\mathrm{T}}$ sum of all $3 \times 3$ calorimeter tower regions. If a $3 \times 3$ region has a summed $E_{\mathrm{T}} 2 \times \mathrm{RMS}$ above the mean of the summed $E_{\mathrm{T}}$ distribution, the central tower is tagged. The $\left\langle E_{\mathrm{T}}\right\rangle$ of cells in each calorimeter sampling layer is computed for 0.1 unit bins in $\eta$ and by excluding cells that are within a cone radius of 0.8 units from any of the tagged towers. The $\eta$-dependence takes into account both physics modifying the underlying event distribution, as well as $\eta$-dependent material in ATLAS. The layer-dependence is important because some layers, especially the last longitudinal layers of the EM and Hadronic calorimeters, have little background to subtract. Tagging high energy regions in the calorimeter reduces the bias of jets on the underlying event $\left\langle E_{\mathrm{T}}\right\rangle$ determination. Once the $\left\langle E_{\mathrm{T}}\right\rangle$ is known, it is subtracted from all of the cells of a given layer and $\eta$ bin. These subtracted cells are used to create new subtracted towers. These subtracted towers are then input to the jet reconstruction algorithm. This is, of course, only one method that could be used to handle the underlying event energy in cone jets. It should be noted that other algorithms are being studied, e.g. $k_{\mathrm{T}}$ and anti- $k_{\mathrm{T}}$ algorithms. The $k_{\mathrm{T}}$ jet algorithm gives similar results to the cone [5] while the anti- $k_{\mathrm{T}}$ study is ongoing.

\section{Differential Jet Studies}

Once full jets are constructed, the goal is to further understand the mechanism of energy loss. One additional advantage of jet reconstruction, compared to single particle and di-hadron 

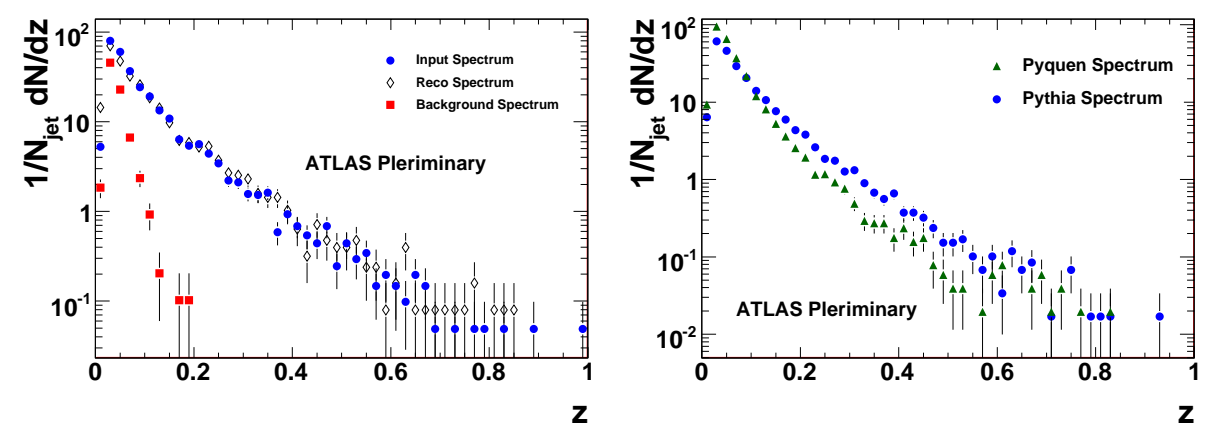

Figure 2: Left: The fragmentation function from input jets and input particles (circles), reconstructed jets and tracks (diamonds), and the fake distribution showing the contribution of the underlying event (squares). The fake distribution is already removed from the reconstructed spectrum. Right: The fragmentation function from PYTHIA (circles) and PYQUEN (triangles) with the ATLAS detector response.

measurements, is the information gained by varying the parameters of the algorithms used. The measurements of jets in heavy ions can be performed as a function of not just centrality and reaction plane but also in the algorithm parameters.

One example of a differential study is measuring the jet $R_{A A}$ as a function of the cone radius $R$. According to a perturbative energy loss model [6], cone jets reconstructed with different $R$ will be sensitive to different amounts of out-of-cone gluon radiation. In Ref. [6] the authors find that binary scaling of the jet cross-section is not recovered until $R=2$, which is probably difficult to measure experimentally. Still the variation of jet $R_{A A}$ as a function of $R$ should be sensitive to the radiated gluon angular distribution, a key prediction of perturbative energy loss models. Figure 1 shows the ratio of reconstructed to input jet spectra as a function of jet $E_{\mathrm{T}}$. The reconstructed spectrum is not yet corrected for any reconstruction inefficiencies or resolutions. This shows that ATLAS will be sensitive to $10 \%$ effects on the jet $R_{A A}$.

Another example is the fragmentation function, $D(z)$, which is the probability for a fragment to have a longitudinal energy fraction $z$ of the jet. The softening of the fragmentation function has long been an expected signature of parton energy loss since the leading, high- $z$, fragments are expected to lose energy, shifting down in $z$ while producing radiated gluons at low $z$ [7]. In ATLAS fragmentation functions are constructed using charged tracks. These charged tracks are constructed in the inner detector with an efficiency of $70 \%$ and fake rates $<1 \%$. The tracks are then extrapolated to the calorimeter, and if the tower to which the track points is in a jet, it is considered a fragment. Only reconstructed tracks with $p_{\mathrm{T}}>2 \mathrm{GeV}$ are considered. The left panel of Figure 2 shows the input PYTHIA fragmentation function compared to the fragmentation function after embedding into HIJING, full reconstruction, and application of a constant efficiency correction. The agreement between input and reconstructed spectra is quite good. This can be compared to the right panel of Figure 2, which shows the softer PYQUEN fragmentation function compared to PYTHIA even after simulating with the ATLAS detector response. We can expect to be sensitive to modifications at this level, a factor of 2 suppression for $z>0.2$, to the fragmentation functions.

Another topic of interest is heavy flavor energy loss. Early expectations from perturbative energy loss was that heavy flavor quarks lose less energy than light flavors. However, this expec- 

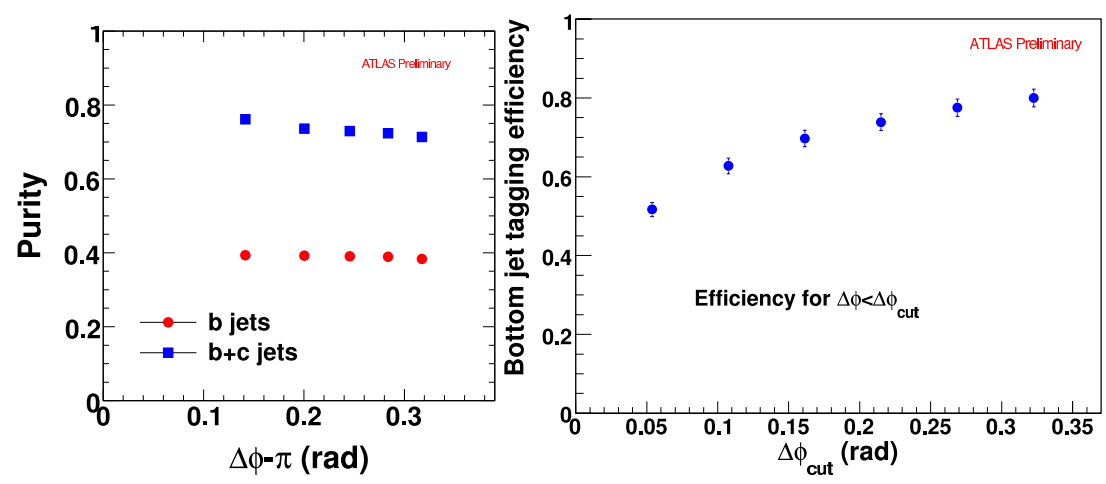

Figure 3: Left: Purity of tagging a bottom jet (circles) and heavy flavor jet (squares) requiring a $>5 \mathrm{GeV}$ muon associated with a jet below $\Delta \phi_{\text {cut }}$. Right: The efficiency of tagging a bottom jet with a given $\Delta \phi_{\text {cut }}$.

tation cannot be currently reconciled with single non-photonic electron data at RHIC [8]. As a first attempt at understanding the ATLAS sensitivity to heavy flavor energy loss, we have studied our ability to tag heavy flavor jets with reconstructed muons. Full simulations of PYTHIA jets embedded into heavy ion events show a correlation in azimuth between heavy flavor (charm and bottom) jets above $70 \mathrm{GeV}$ and reconstructed muons above $5 \mathrm{GeV}$. The left panel of Figure 3 shows the purity for tagging a heavy flavor jet or bottom jet as a function of the azimuthal angle cut imposed. The right panel shows the efficiency for tagging bottom jets using the muon. For $\Delta \phi_{\text {cut }}<0.15 \mathrm{rad}$, the bottom jet tagging efficiency is $70 \%$ with a purity of $40 \%$ for b jets. Further techniques, such as reconstructing a displaced vertex associated with the jet, can be used to improve these numbers.

\section{Summary}

In this contribution we briefly outlined the program for full jet reconstruction in heavy ion collisions with the ATLAS detector. Full jet reconstruction not only opens up new measurements, such as the jet $R_{A A}$ and fragmentation functions $D(z)$, but it also provides new information, through the variation of the jet reconstruction parameters for differential studies of energy loss on both light and heavy flavor jets. With its large acceptance tracking, calorimetry, and muon spectrometer, ATLAS is poised to make important contributions to the understanding of parton energy loss through the study of jets in heavy ion collisions at the LHC.

\section{References}

[1] J. Nagle, these proceedings A. Sickles, these proceedings

[2] S. Salur, these proceedings

[3] The ATLAS Experiment at the CERN Large Hadron Collider G. Aad, et al. (ATLAS Collaboration) JINST 3, (2008) S08003

[4] ATLAS Heavy Ion Physics Letter of Intent, CERN-LHCC-2004-009

[5] N. Grau (ATLAS Collaboration) arXiv:0805.4656 [nucl-ex]

[6] I. Vitev, S. Wicks, and B. Zhang JHEP 11 (2008) 093

[7] N. Armesto et al. JHEP 02 (2008) 048

[8] A. Adare et al. (PHENIX Collaboration), Phys. Rev. Lett. 98, 172301 (2007) 